\title{
Tailoring Non-Abelian Gauge Theory for Digital Quantum Simulation
}

\author{
Jesse Stryker*† \\ Institute for Nuclear Theory (INT), University of Washington, Seattle, WA 98195, USA \\ E-mail: stryker@uw.edu

\section{Indrakshi Raychowdhury} \\ Maryland Center for Fundamental Physics (MCFP) and Department of Physics, University of \\ Maryland, College Park, MD 20742, USA \\ E-mail: iraychow@umd.edu
}

\begin{abstract}
The question of how to efficiently formulate Hamiltonian gauge theories is experiencing renewed interest due to advances in building quantum simulation platforms. We introduce a reformulation of an SU(2) Hamiltonian lattice gauge theory - a loop-string-hadron (LSH) formulation-in which the dynamical degrees of freedom are localized pieces of flux loops, meson strings, and hadrons. LSH operators are first derived from Schwinger bosons and used to construct a Hilbert space with the non-Abelian Gauss law built into it. They are subsequently factored into products of "normalized" ladder operators and diagonal matrices, priming them for classical or quantum information processing. The LSH formalism alleviates several disadvantages of quantumsimulating the Kogut-Susskind formulation and makes little use of structures specific to SU(2). Its conceptual clarity makes it an attractive approach to apply to other non-Abelian groups like SU(3).
\end{abstract}

37th International Symposium on Lattice Field Theory - Lattice2019

16-22 June 2019

Wuhan, China

\footnotetext{
* Speaker.

$\dagger$ JRS gratefully acknowledges the Local Organizing Committee for financial support to attend Lattice 2019.
} 


\section{Introduction}

Lattice quantum field theory, in particular lattice QCD, has proven to be a successful nonperturbative approach to studying gauge theories numerically. Lattice field theory calculations are usually performed by importance-sampling the functional integral in Euclidean space (imaginary time). These computational feats have predominantly characterized static or equilibrium properties at zero chemical potential [1]. However, lattice QCD calculations with non-zero baryon chemical potential, with a topological $\theta$-term, or in real (Minkowskian) time are generally hampered by exponentially hard sign problems. The fact that these scenarios can be so much harder is an artifact of the way they are formulated for simulation on classical machines, i.e., the path integral and the breakdown of Monte Carlo methods when applied to it. A class of problems that especially stands to benefit from new methods is real-time dynamics.

The Hamiltonian formulation of gauge theories [2], which requires singling out a timelike direction, seems most natural for describing intrinsically real-time processes. In the 1980s, it was proposed that computers based on quantum mechanical degrees of freedom ought to be better suited for simulating Hamiltonian dynamics of quantum many-body systems [3], such as a gauge theory. The current arrival of functional quantum devices [4] thus creates an urgent need to know how the structure of a Hamiltonian lattice gauge theory can be related to that of quantum architectures. In this work, we revisit and reformulate a non-Abelian lattice gauge theory-SU(2) gauge theory in $1+1$ dimensions with one flavor of staggered quarks - ultimately putting it into an explicit form to which (classical or) quantum algorithms can be readily applied: a loop-string-hadron (LSH) formulation of SU(2) lattice gauge theory, which uses local loops, strings, and hadrons as dynamical variables. For a more in-depth treatment, including transcription of the Hamiltonian and higher spatial dimensions, the reader may consult Ref. [5].

This LSH formulation follows from working with strictly SU(2)-invariant operators and is an extension of the Schwinger boson (prepotential) formulation of lattice gauge theory $[6,7,8]$. The non-Abelian Gauss law that usually appears as a constraint is made intrinsic, meaning the local excitations are physical and even intuitive. The price paid is the introduction of an Abelian Gauss law that must be enforced instead, and the introduction of additional lattice links. These are not fundamental hurdles because (i) the Abelian constraints are simpler to work with, and (ii) if the Abelian constraints are also solved, then the gauge-invariant Hilbert space is covered much more efficiently than it would be in a Kogut-Susskind formulation. (Addressing the latter point is the subject of ongoing work.) Importantly, by making the operator structure so explicit, algorithms can start being applied to simulating dynamics and compared against any other proposals made for nonAbelian simulations. In a related work [9], we provide a mapping of the LSH formalism to qubits along with quantum circuit solutions to all the constraints, representing a major advance towards implementing verifiably-gauge-invariant states and quantum error mitigation. Generalizing the present LSH formalism to SU(3) would be a key step towards future applications to lattice QCD.

\section{Schwinger boson formulation}

The Schwinger boson formulation of lattice gauge theory realizes electric fields $E$ and link operators $U$ with bilinears of bosonic harmonic oscillator doublets $a_{\alpha}(L / R)$ associated with the left 
$(L)$ and right $(R)$ ends of each spatial link $(x, i)$. (Time is continuous.) The $\mathrm{SU}(2)$ electric fields on a link are constructed as $E_{L / R}^{\mathrm{a}} \equiv a^{\dagger}(L / R) T^{\mathrm{a}} a(L / R)$, where $T^{\mathrm{a}}=\sigma^{\mathrm{a}} / 2$. Beyond the $\mathrm{SU}(2)_{L / R}$ electric fields, there also exist $\mathrm{U}(1)$ generators: $N_{L / R}=a^{\dagger}(L / R) \cdot a(L / R)$. To be equivalent to the KogutSusskind formulation [2], the left and right Casimirs $E^{\mathrm{a}} E^{\mathrm{a}}$ must be equal, leading to an Abelian Gauss law (AGL) relating the U(1) generators along each link: $N_{L}(x, i) \mid$ phys $\rangle=N_{R}\left(x+e_{i}, i\right) \mid$ phys $\rangle$. As for the $\mathrm{SU}(2)$ link operators, these are given by $U \equiv U_{L} U_{R}$, with

$$
U_{L} \equiv \frac{1}{\sqrt{N_{L}+1}}\left(\begin{array}{cc}
a_{2}^{\dagger}(L) & a_{1}(L) \\
-a_{1}^{\dagger}(L) & a_{2}(L)
\end{array}\right), \quad U_{R} \equiv\left(\begin{array}{cc}
a_{1}^{\dagger}(R) & a_{2}^{\dagger}(R) \\
-a_{2}(R) & a_{1}(R)
\end{array}\right) \frac{1}{\sqrt{N_{R}+1}} .
$$

In this work, we additionally consider a (staggered) fermionic matter field $\psi(x)=\left(\psi_{1}(x), \psi_{2}(x)\right)$. Physically-permissible wave functions must be annihilated by the Schwinger boson implementations of the non-Abelian Gauss law operators, $G^{\mathrm{a}}(x)=\sum_{i}\left(E_{L, i}^{\mathrm{a}}(x)+E_{R, i}^{\mathrm{a}}(x)\right)+\psi(x)^{\dagger} T^{\mathrm{a}} \psi(x)$.

The gauge field Hilbert space is built from the local bosonic oscillators, two modes at the left and right ends of every link. The natural quantum numbers for such a Hilbert space would be the occupancies, $\left\{N_{1}(L), N_{2}(L), N_{1}(R), N_{2}(R)\right\}$ for each link. This is equivalent to an "angular momentum" basis that diagonalizes, say, $\left\{E_{L}^{\mathrm{a}} E_{L}^{\mathrm{a}}, E_{L}^{z}, E_{R}^{\mathrm{a}} E_{R}^{\mathrm{a}}, E_{R}^{z}\right\}$, but the spectrum of quantum numbers is different. Truncating the Kogut-Susskind theory at some angular momentum $j_{\max }$, $E^{\mathrm{a}} E^{\mathrm{a}} \leq j_{\max }\left(j_{\max }+1\right)$, is equivalent to truncating all Schwinger boson occupancies at $j_{\max }$.

Compared to an angular momentum basis, the Schwinger boson formulation offers the following features that are promising for developing quantum algorithms: (i) Symmetric quantum numbers: All quantum numbers are structured identically. This makes it obvious how one could represent these quantum numbers with binary registers. It is also obvious how to truncate the electric field (a uniform cutoff on all the occupation numbers). (ii) Non-group-specific matrix elements: The link operator is expressed in terms of simple harmonic oscillator ladder operators, and Clebsch-Gordon coefficients are implicit in the various rescaling factors carried along by them. In this sense, the elementary degrees of freedom are group-agnostic. (Of course, in going from SU(2) to $\mathrm{SU}(3)$ one needs $\mathrm{SU}(3)$ irreducible Schwinger bosons [10].)

The preceding points unfortunately say little about how to deal with the non-Abelian constraints or about redundancy of states. To remedy this, we note that local gauge transformations act site-locally, with Schwinger bosons and matter all transforming identically. This enables one to construct site-local intertwining operators automatically invariant under the action of the local generators; these can be identified as segments of all possible SU(2)-invariant excitations hosted by a site (such as a section of a Wilson loop). Using these, one can construct an SU(2)-invariant Hilbert space locally at each site. In pure gauge theory, the resulting local "loop states" $[11,12]$ are characterized by integer-valued loop quantum numbers directly related to the angular momentum flux $j$. Truncating the Kogut-Susskind theory at some representation $j_{\max }$ then becomes equivalent to truncating local loop numbers at $2 j_{\max } /(2 d-1)$.

A drawback of the loop basis is that it is overcomplete. Finding the complete and orthogonal gauge-invariant Hilbert space requires solving the Mandelstam constraints, which becomes increasingly complicated in higher dimensions and with higher cutoff. These issues have been discussed in great detail in earlier works on the prepotential formulation of pure gauge theory [13]. A central objective of the loop-string-hadron framework below will be to give a complete and lo- 
cal description of gauge-invariant dynamics with minimal redundancy, equipped with fundamental matter, and adaptable to any number of spatial dimensions.

\section{Loop-string-hadron formulation: One dimension}

We now derive a loop-string-hadron formulation starting from prepotentials that has nonAbelian gauge invariance built into it. We focus on $1+1$ dimensions, where the essential features of coupling to matter-which was not previously a part of the prepotential framework-already appear. (For more details, see Ref. [5].) Each site $x$ of this lattice is connected to one incoming link along direction $i$ and one outgoing link along direction $o$, associated with Schwinger boson doublets $\hat{a}_{\alpha}(R)$ and $\hat{a}_{\alpha}(L)$, respectively. A staggered fermion field $\hat{\psi}=\left(\hat{\psi}_{1}, \hat{\psi}_{2}\right)$ lives on the sites.

The site-local doublets can contract in many possible ways to form SU(2) singlets. The complete set of SU(2) invariants at a 1d site is obtained by constructing all possible singlet tensors out the available doublets and their conjugates. The unitary equivalence of fundamental and antifundamental doublets in $\mathrm{SU}(2)$ means $\tilde{a}_{\alpha}^{\dagger}(L / R) \equiv \varepsilon_{\alpha \beta} a_{\beta}^{\dagger}(L / R)$ provide another set of doublets to work with. Using the available tensors, the complete set of non-vanishing singlets is given as follows.

$$
\begin{array}{rlrl}
\mathscr{L}^{++} & =a(R)_{\alpha}^{\dagger} a(L)_{\beta}^{\dagger} \varepsilon_{\alpha \beta} & & \mathscr{L}^{--}=a(R)_{\alpha} a(L)_{\beta} \varepsilon_{\alpha \beta}=\left(\mathscr{L}^{++}\right)^{\dagger} \\
\mathscr{L}^{+-} & =a(R)_{\alpha}^{\dagger} a(L)_{\beta} \delta_{\alpha \beta} & & \mathscr{L}^{-+}=a(R)_{\alpha} a(L)_{\beta}^{\dagger} \delta_{\alpha \beta}=\left(\mathscr{L}^{+-}\right)^{\dagger} \\
\mathscr{S}_{\text {in }}^{++} & =a(R)_{\alpha}^{\dagger} \psi_{\beta}^{\dagger} \varepsilon_{\alpha \beta} & & \mathscr{S}_{\text {in }}^{--}=a(R)_{\alpha} \psi_{\beta} \varepsilon_{\alpha \beta}=\left(\mathscr{S}_{\text {in }}^{++}\right)^{\dagger} \\
\mathscr{S}_{\text {in }}^{+-}=a(R)_{\alpha}^{\dagger} \psi_{\beta} \delta_{\alpha \beta} & \mathscr{S}_{\text {in }}^{-+}=a(R)_{\alpha} \psi_{\beta}^{\dagger} \delta_{\alpha \beta}=\left(\mathscr{S}_{\text {in }}^{+-}\right)^{\dagger} \\
\mathscr{S}_{\text {out }}^{++}=\psi_{\alpha}^{\dagger} a(L)_{\beta}^{\dagger} \varepsilon_{\alpha \beta} & \mathscr{S}_{\text {out }}^{--}=\psi_{\alpha} a(L)_{\beta} \varepsilon_{\alpha \beta}=\left(\mathscr{S}_{\text {out }}^{++}\right)^{\dagger} \\
\mathscr{S}_{\text {out }}^{+-}=\psi_{\alpha}^{\dagger} a(L)_{\beta} \delta_{\alpha \beta} & \mathscr{S}_{\text {out }}^{-+}=\psi_{\alpha} a(L)_{\beta}^{\dagger} \delta_{\alpha \beta}=\left(\mathscr{S}_{\text {out }}^{+-}\right)^{\dagger} \\
\mathscr{H}^{++}=-\frac{1}{2 !} \psi_{\alpha}^{\dagger} \psi_{\beta}^{\dagger} \varepsilon_{\alpha \beta} & \mathscr{H}^{--}=\frac{1}{2 !} \psi_{\alpha} \psi_{\beta} \varepsilon_{\alpha \beta}=\left(\mathscr{H}^{++}\right)^{\dagger}
\end{array}
$$

$\mathscr{L}^{\sigma, \sigma^{\prime}}$ are pure gauge loop operators, $\mathscr{S}_{\text {in }}^{\sigma, \sigma^{\prime}}$ are incoming string operators, $\mathscr{S}_{\text {out }}^{\sigma, \sigma^{\prime}}$ are outgoing string operators, and $\mathscr{H}^{\sigma, \sigma}$ are hadron operators. In addition, there are gauge flux and quark number operators: $\mathscr{N}_{L / R}=a(L / R)_{\alpha}^{\dagger} a(L / R)_{\alpha}$ and $\mathscr{N}_{\psi}=\psi_{\alpha}^{\dagger} \psi_{\alpha}$. These invariants exhaust all possible singlet bilinears and they are referred to as LSH operators.

By working solely with $\mathrm{SU}(2)$ singlets, the only gauge constraints that will have to be enforced 'by hand' are the Abelian Gauss Laws: $\left(\mathscr{N}_{R}(x+1)-\mathscr{N}_{L}(x)\right) \mid$ phys $\rangle=0$. This was always the case in the Schwinger boson formulation, but now all available operators (replacing $E, U$, and $\psi$ ) have the on-site non-Abelian Gauss law intrinsically solved. Importantly, the remaining constraints all commute. These AGL constraints retain the same form they had in pure gauge loop formulations [12]; one could go further by solving them too, but here the map will be given just for passing to $\mathrm{SU}(2)$-invariant variables.

To prepare for computational algorithms, it is helpful to set up a basis. As will be shown below, one can enumerate states directly in terms of SU(2)-invariant LSH excitations-leading to a 'loopstring-hadron basis.' In this way, only physically-allowable on-site states are ever represented.

A second practical issue to be addressed concerns operator factorization. LSH operators change state normalization in addition to changing quantum numbers. Factorizing these two behaviors has the benefits of making the matrix elements of any operator completely evident and also 
setting the stage for a Wigner-Jordan transformation. This factorization will be done for convenience with respect to a LSH basis (though the factorization itself is basis-independent).

A 1d lattice site hosts three SU(2)-invariant degrees of freedom corresponding to the original occupation numbers $\mathscr{N}_{L / R}$, and $\psi^{\dagger} \psi$. However, these are constrained by the possible kinds of excitations that LSH operators produce. A more physical site-local characterization would instead describe the configuration in terms of allowable SU(2)-invariant excitations. Such a loop-stringhadron basis of unnormalized kets, denoted by a double-bar ket $\|\rangle$, can be defined as follows:

$$
\begin{array}{rlrl}
\left.\| n_{l}, n_{i}=0, n_{o}=0\right\rangle & \equiv\left(\mathscr{L}^{++}\right)^{n_{l}}|0\rangle, & \left.\| n_{l}, n_{i}=0, n_{o}=1\right\rangle \equiv\left(\mathscr{L}^{++}\right)^{n_{l}} \mathscr{S}_{\text {out }}^{++}|0\rangle \\
\left.\| n_{l}, n_{i}=1, n_{o}=0\right\rangle \equiv\left(\mathscr{L}^{++}\right)^{n_{l}} \mathscr{S}_{\text {in }}^{++}|0\rangle, & \left.\| n_{l}, n_{i}=1, n_{o}=1\right\rangle \equiv\left(\mathscr{L}^{++}\right)^{n_{l}} \mathscr{H}^{++}|0\rangle
\end{array}
$$

where $n_{l}=(0,1,2, \cdots), n_{i}=(0,1), n_{o}=(0,1)$, the local vacant state $|0\rangle$ is annihilated by any LSH operator carrying at least one minus sign, and $\langle 0 \mid 0\rangle=1 . n_{l}$ counts the units of electric flux running through the site. $n_{i}$ and $n_{o}$ indicate quark content, which may be accompanied by the end of a flux string for gauge invariance. The corresponding unit-normalized basis is given by $\left.\left|n_{l}, n_{i}, n_{o}\right\rangle=\left(n_{l} !\left(n_{l}+1+\left(n_{i}+n_{o} \bmod 2\right)\right) !\right)^{-1 / 2}|| n_{l}, n_{i}, n_{o}\right\rangle$. The number operators appropriate for this basis are related to the previous ones by

$$
\begin{aligned}
\mathscr{N}_{i} & \equiv \frac{1}{2}\left[\mathscr{N}_{\psi}+\mathscr{N}_{R}-\mathscr{N}_{L}\right], \\
\mathscr{N}_{o} & \equiv \frac{1}{2}\left[\mathscr{N}_{\psi}+\mathscr{N}_{L}-\mathscr{N}_{R}\right], \\
\mathscr{N}_{l} & \equiv \frac{1}{2}\left[\mathscr{N}_{L}+\mathscr{N}_{R}-\mathscr{N}_{\psi}+\frac{1}{2}\left(\mathscr{N}_{\psi}^{2}-\left(\mathscr{N}_{L}-\mathscr{N}_{R}\right)^{2}\right)\right] .
\end{aligned}
$$

Dynamics ultimately couples sites and is expressed using states of the lattice as a whole. It is not necessary to prescribe how the lattice Hilbert space is constructed from the on-site Hilbert spaces to address the Abelian Gauss laws required of physical lattice states: using the $\left|n_{l}, n_{i}, n_{o}\right\rangle$ on-site bases, this translates to $\left[n_{l}+n_{o}\left(1-n_{i}\right)\right]_{x}=\left[n_{l}+n_{i}\left(1-n_{o}\right)\right]_{x+1}$.

The on-site operators can now be factored as discussed earlier, at which point matrix elements with respect to the LSH basis can be read off easily. Pertaining to the loop number $n_{l}$, we first introduce normalized ladder operators, $\Lambda^{+}$and $\Lambda^{-}$:

$$
\Lambda^{ \pm} \equiv \mathscr{L}^{ \pm \pm}\left[\left(\mathscr{N}_{l}+\frac{1}{2} \pm \frac{1}{2}\right)\left(\mathscr{N}_{l}+\frac{3}{2} \pm \frac{1}{2}+\left(\mathscr{N}_{i} \oplus \mathscr{N}_{o}\right)\right)\right]^{-1 / 2}
$$

Here a "normalized operator" refers to any operator $\mathscr{O}$ such that non-vanishing eigenvalues of $\mathscr{O}^{\dagger} \mathscr{O}$ are unity. The $\Lambda^{ \pm}$operators move states up or down the ladder of $n_{l}$ without changing normalization, except for the possibility of annihilation at the bottom of the ladder. As for the quark quantum numbers, these are affected by the string operators (and the mixed-type loop operators $\mathscr{L}^{ \pm, \mp}$ ). The string operators obey fermion-like anticommutation relations, but they are not canonically normalized. This motivates introducing SU(2)-invariant fermionic modes $\chi_{i / o}$ to describe them, with $\left\{\chi_{q^{\prime}}, \chi_{q}\right\}=\left\{\chi_{q^{\prime}}^{\dagger}, \chi_{q}^{\dagger}\right\}=0$ and $\left\{\chi_{q^{\prime}}, \chi_{q}^{\dagger}\right\}=\delta_{q^{\prime} q}$. These also qualify as normalized ladder operators. Because string operators can affect loop numbers, it will prove helpful to also introduce the following shorthand conditional ladder operators that act differently on the two eigenspaces of $\mathscr{N}_{q}$ :

$$
\left(\Lambda^{ \pm}\right)^{\mathscr{N}_{q}} \equiv\left(1-\mathscr{N}_{q}\right)+\Lambda^{ \pm} \mathscr{N}_{q}, \quad\left(\Lambda^{ \pm}\right)^{1-\mathscr{N}_{q}} \equiv \Lambda^{ \pm}\left(1-\mathscr{N}_{q}\right)+\mathscr{N}_{q} . \quad(q=i, o)
$$


Equipped with the normalized ladder operators, all LSH operators factorize as follows.

$$
\begin{aligned}
& \mathscr{L}^{++}=\Lambda^{+} \sqrt{\left(\mathscr{N}_{l}+1\right)\left(\mathscr{N}_{l}+2+\left(\mathscr{N}_{i} \oplus \mathscr{N}_{o}\right)\right)} \\
& \mathscr{L}^{+-}=-\chi_{i}^{\dagger} \chi_{o} \\
& \mathscr{S}_{\text {in }}^{++}=\chi_{i}^{\dagger}\left(\Lambda^{+}\right)^{\mathscr{N}_{o}} \quad \sqrt{\mathscr{N}_{l}+2-\mathscr{N}_{o}} \\
& \mathscr{S}_{\text {out }}^{++}=\chi_{o}^{\dagger}\left(\Lambda^{+}\right)^{\mathscr{N}_{i}} \sqrt{\mathscr{N}_{l}+2-\mathscr{N}_{i}} \\
& \mathscr{S}_{\text {in }}^{-+}=\chi_{o}^{\dagger}\left(\Lambda^{-}\right)^{1-\mathscr{N}_{i}} \sqrt{\mathscr{N}_{l}+2 \mathscr{N}_{i}} \\
& \mathscr{S}_{\text {out }}^{+-}=\chi_{i}^{\dagger}\left(\Lambda^{-}\right)^{1-\mathscr{N}_{o}} \sqrt{\mathscr{N}_{l}+2 \mathscr{N}_{o}} \\
& \mathscr{H}^{++}=\chi_{i}^{\dagger} \chi_{o}^{\dagger}
\end{aligned}
$$

$$
\begin{aligned}
& \mathscr{L}^{--}=\Lambda^{-} \sqrt{\mathscr{N}_{l}\left(\mathscr{N}_{l}+1+\left(\mathscr{N}_{i} \oplus \mathscr{N}_{o}\right)\right)} \\
& \mathscr{L}^{-+}=\chi_{i} \chi_{o}^{\dagger} \\
& \mathscr{S}_{\text {in }}^{--}=\chi_{i}\left(\Lambda^{-}\right)^{\mathscr{N}_{o}} \sqrt{\mathscr{N}_{l}+2\left(1-\mathscr{N}_{o}\right)} \\
& \mathscr{S}_{\text {out }}^{--}=\chi_{o}\left(\Lambda^{-}\right)^{\mathscr{N}_{i}} \sqrt{\mathscr{N}_{l}+2\left(1-\mathscr{N}_{i}\right)} \\
& \mathscr{S}_{\text {in }}^{+-}=\chi_{o}\left(\Lambda^{+}\right)^{1-\mathscr{N}_{i}} \sqrt{\mathscr{N}_{l}+1+\mathscr{N}_{i}} \\
& \mathscr{S}_{\text {out }}^{-+}=\chi_{i}\left(\Lambda^{+}\right)^{1-\mathscr{N}_{o}} \sqrt{\mathscr{N}_{l}+1+\mathscr{N}_{o}} \\
& \mathscr{H}^{--}=-\chi_{i} \chi_{o}
\end{aligned}
$$

The factorizations are all expressed with diagonal scaling operators sitting on the right and normalized ladder operators following to the left. Noting that $\left|n_{l}, n_{i}, n_{o}\right\rangle=\left(\Lambda^{+}\right)^{n_{l}}\left(\chi_{i}^{\dagger}\right)^{n_{i}}\left(\chi_{o}^{\dagger}\right)^{n_{o}}|0\rangle$, one can easily see how acting an LSH operator on a basis state rescales it and changes its quantum numbers.

To apply these factorizations in the context of digital quantum simulation, one needs to map the fermionic modes into commuting computational degrees of freedom. To guide this, we first look toward how the fermionic operators are used in the time evolution operator. The fermionic modes $\chi_{i}(x), \chi_{o}(x)$ of a length $L_{x}$ lattice $\left(x=0, \ldots, L_{x}-1\right)$ express physical (SU(2)-invariant) quark degrees of freedom that are dynamically coupled through "hopping terms." However, it turns out that the $\chi_{i}$ 's and $\chi_{o}$ 's decouple from each other in the staggered Hamiltonian. For example, $\mathscr{S}_{\text {out }}^{+\sigma}(x) \mathscr{S}_{\text {in }}^{\sigma-}(x+1)$ are hopping terms whose fermionic operator content takes the form

$$
\mathscr{S}_{\text {out }}^{++}(x) \mathscr{S}_{\text {in }}^{+-}(x+1) \sim \chi_{o}^{\dagger}(x) \chi_{o}(x+1) \cdots, \quad \mathscr{S}_{\text {out }}^{+-}(x) \mathscr{S}_{\text {in }}^{--}(x+1) \sim \chi_{i}^{\dagger}(x) \chi_{i}(x+1) \cdots .
$$

We therefore relabel the fermionic modes of the lattice using $\Psi_{k}$ for $k=0, \ldots, 2 L_{x}-1$, with

$$
\Psi_{k}=\left\{\begin{array}{ll}
\chi_{i}(k), & 0 \leq k \leq L_{x}-1 \\
\chi_{o}\left(k-L_{x}\right), & L_{x} \leq k \leq 2 L_{x}-1
\end{array} .\right.
$$

The Wigner-Jordan transformation converts the $\Psi_{k}$ into spin operators via $\Psi_{k} \equiv \sigma_{k}^{+} \prod_{k^{\prime}=0}^{k-1} \sigma_{k^{\prime}}^{z}$. Assuming open boundary conditions, all fermionic couplings are then nearest-neighbor in the $x$ coordinate as well as the $k$ label. The couplings in the hopping terms take the final form $\sigma_{k}^{ \pm} \sigma_{k+1}^{\mp}$ :

$$
\chi_{i}^{\dagger}(x) \chi_{i}(x+1)=\sigma_{x}^{-} \sigma_{x+1}^{+}, \quad \chi_{o}^{\dagger}(x) \chi_{o}(x+1)=\sigma_{L_{x}+x}^{-} \sigma_{L_{x}+x+1}^{+} .
$$

Hence, as far as the staggered Hamiltonian for a $1 \mathrm{~d}$ open lattice is concerned, it is possible to essentially replace anticommuting $\chi$ 's and $\chi^{\dagger}$ 's with commuting $\sigma^{+}$'s and $\sigma^{-}$'s in the operator factorizations.

\section{Conclusions}

Schwinger bosons were used to construct all manifestly SU(2)-invariant loop-string-hadron operators. These operators were then used to construct an LSH basis in which every possible combination of quantum numbers describes a unique set of allowable on-site excitations. The remaining constraints, Abelian Gauss laws, impose a simple criterion that definitively separates LSH basis 
states into allowed and unallowed sectors. Looking toward applications, all LSH operators were factored for convenience with respect to that basis (and leaving no explicit reference to SU(2)).

In $1 \mathrm{~d}$ with open boundary conditions, one can essentially replace fermionic physical-quark operators with spin operators. The local bases and operator factorizations carry over to "matter sites" in $d>1$, although the simplicity of the qubit mapping for quark modes is not as straightforward.

A far more expansive discussion of this work can be found in Ref. [5], including complete translations of the Hamiltonian in $d=(1,2,3)$ spatial dimensions.

\section{Acknowledgments}

IR is supported by the U.S. Department of Energy (DOE), Office of Science, Office of Advanced Scientific Computing Research (ASCR) Quantum Computing Application Teams program, under fieldwork proposal number ERKJ347, and by the MCFP. JRS was supported by DOE Grant No. DE-FG02-00ER41132, and by the National Science Foundation Graduate Research Fellowship under Grant No. 1256082. Part of this research was carried out with support from a Thomas L. and Margo G. Wyckoff Endowed Faculty Fellowship.

\section{References}

[1] A. S. Kronfeld, Twenty-first century lattice gauge theory: Results from the quantum chromodynamics Lagrangian, Annu. Rev. Nucl. Part. Sci. 62 (2012) 265.

[2] J. Kogut and L. Susskind, Hamiltonian formulation of Wilson's lattice gauge theories, Phys. Rev. D 11 (1975) 395.

[3] R. P. Feynman, Simulating physics with computers, Int. J. Theor. Phys. 21 (1982) 467.

[4] F. Arute, K. Arya, R. Babbush, D. Bacon, J. C. Bardin, R. Barends et al., Quantum supremacy using a programmable superconducting processor, Nature 574 (2019) 505.

[5] I. Raychowdhury and J. R. Stryker, Loop, String, and Hadron Dynamics in SU(2) Hamiltonian Lattice Gauge Theories, arXiv:1912.06133 [hep-lat, physics:hep-th, physics:quant-ph] (2019) [1912.06133].

[6] J. Schwinger, On angular momentum, Tech. Rep. NYO-3071, U.S. Atomic Energy Commission, Oak Ridge, Tennessee, Jan., 1952.

[7] M. Mathur, Harmonic oscillator pre-potentials in SU (2) lattice gauge theory, J. Phys. A: Math. Gen. 38 (2005) 10015.

[8] M. Mathur, I. Raychowdhury and R. Anishetty, $S U(N)$ irreducible Schwinger bosons, Journal of Mathematical Physics 51 (2010) 093504.

[9] I. Raychowdhury and J. R. Stryker, Solving Gauss's Law on Digital Quantum Computers with Loop-String-Hadron Digitization, arXiv:1812.07554 [hep-lat, physics:hep-th, physics:quant-ph] (2020) [1812.07554].

[10] R. Anishetty, M. Mathur and I. Raychowdhury, Irreducible SU(3) Schwinger bosons, Journal of Mathematical Physics 50 (2009) 053503.

[11] M. Mathur, Loop states in lattice gauge theory, Physics Letters B 640 (2006) 292.

[12] M. Mathur, Loop approach to lattice gauge theories, Nucl. Phys. B 779 (2007) 32.

[13] I. Raychowdhury, Low energy spectrum of SU(2) lattice gange theory, Eur. Phys. J. C 79 (2019) 235. 